\title{
LINEAR OSCILLA'TIONS OF A DROP IN UNIFORM ALTERNATING ELECTRIC FIELDS
}

\author{
Wenrui Yang and Thomas E. Carleson \\ Department of Chemical Engineering \\ University of Idaho \\ Moscow, Idaho 83843
}

Prepared for presentation at AIChE 1990 Annual Meeting. November, 1990

Electric Charges at Interfaces

Ortober, 1990

\author{
AIChE shall not be responsible for statements or opinions \\ contained in papers or printed in its publicarions
}

\section{DISCLAIMER}

This report was prepared as an account of work sponsored by alt agency of the Uniled Stutes Government. Neithet the United States Government nor any agency therenf, nor any of their employees, makes any warranty, express or implied, of assumes any legal liability or responsibility for the accuracy, completeness, or usefulness of any information, apparatus, product, of process disclosed, or represents that its use would not infringe privatcly owned rights. Refer. ence herein to any specific commercial product, process, or service by trade name, tradernark, manufacturer, or otherwise does not necessarily constitute or imply its endorsemenl, recom. mendation, or favoring by the United States Government or any agency therent. The views and opinions of authors expressed herein do not mecessarily state or reflect those of the United States Government of sny agency thereof. \section{MASTER}


Oscillations of a conducting drop inmersed in a dielectric fluid in an alternating electric field has been modelled in order to understand the enhancement of the transport processes by the electric field. Numerical solutions for oscillation amplitude, velocity distribution, resonant frequency and streamlines were obtained. The effects of viscosity and density on the resonant frequency and the velocity distribution were investigated. It was found that the resonant frequency of viscous fluids was always smaller than the free oscillation frequency of the same droplet. The predicted scanning. frequency response curve and the streamlines agree well with the experimental observations. 
Introduction

Applications of electric force in direct contact heat transfer process or liquid extraction can result in a better energy efficiency than the conventional agitation methods (Thornton 1968). For liquid systems, which have insulating continuous phases and conducting dispersed phases, an imposed electric fielo will disturb the interface by interacting with induced electric charge, and thus enhance mass or heat transfer race. In a static field, the electric force can reduce drop size, increase drop velocity, therefore, increase the interfacial. area and the transport coefficient. An altemating electric field can also reduce the drop size effectively (Kawalski and ziolkowski. 1981). Experiments on direct contact heat transfer showed that an alternating electric field with a proper frequency was more efficient than a static field in enhancing the heet transfer coefficient (Kaji er al., 1980, 1985). The expericents also showed that the enhancement of the heat transfer coefficient was directly related to the drop oscillations induced by the electric force. To understano the effects of the electric fields on the transport processes, we need knowledge of the hydrodymamics of the electrically forced drop oscillations.

Studies on free oscillations of a drop (Miller and Scriven 1968; Prosperetti 1980; Marston 1980) have revealed that a drop oscillates at its characteristic frequencies no matter how the oscfllations are excited. For viscous fluids, the oscillation amplitudes decay gradually due to viscous dissipation. Drop oscillations under the sustained action of an external alternating force are different. The oscillation frequencies are the sare as those of the excernal force (Lanb 1945), but the amplitudes are functions of the external forces as 
well as properties of the fluid system. A quasi-steady external force matritains the amplitudes constant, therefore, the decay factors are zero. Torza et al. (1971) and Sozou (1972) have investigated oscillations of an uncharged crop in alternating electric fields. The attention of their work was paid to the effects of electric properties (resistivity and dielectric constant) on the drop deformation and the flow patterns. They did not discuss the effects of the hydrodynamic properties, which are more essential to the study of the transport processes. Drop oscillations driven by acoustic waves have been studied by Marston (1980). Explicit solutions for oscillation amplitudes and phase shift angles were obtained for low viscosity systems. For solutions in a wide viscosity range, numerical methods must be used.

In this paper, we present a model for the hydrodynanics of small amplitude oscillations of a conducting drop immersed in a dielectric fluid in an alternating electric field. Expressions of velocity distributions are obtained analytically and boundary condition equations are solved numericaliy. Resonan: frequencies are predicted and effects of viscosity and density on Flow fields are discussed.

Model

Consider a charged fluid sphere immersed in another imiscible fluid. Both fluids are assumed incompressible and Newtonian, and properties in each phase are uniform. The interface is presumed to be free from any contanination by surfactants. It is also assumed that the effect of gravity on drop deformation can be neglected ( $g R^{2} \Delta \rho / \sigma$ is small). Only small amplicude oscillations are 
considered, therefore the nonlinear term in the Navier-Stokes equation can be neglected (Levich 1962). It is further assumed that electric field far from the drop is uniform, the drop phase is corductive and the concinuous phase dielectric so that electric equilibrium can be reached instantly and charge loss negligible. This study is limited to the axisymetric flow, which has been frequently observed in experiments. Spherical coordinates $(r, \theta, \phi)$ will be used and the origin is at the drop center. $\theta-0$ is taken as the symraetry axis (the electric field is in this direction). The momentum equation is

$$
\frac{\partial v}{\partial t}-\nu \nabla^{2} v \cdot \frac{1}{\rho}-\nabla p
$$

The continuity equation for an incompressible fluid is

$$
\nabla \cdot v-0
$$

By taking the divergence of the momentum equation and subtracting the continuity" equation, the following pressure equation is obtained (Chandrasekhar 1961).

$$
\nabla^{2} p=0
$$

The boundary conditions include: continuity of the normal and tangentiai velocities at the interface; normal stress balance at the interface: $\varphi_{:}+_{3}$. $T_{r o}$; continuity of the tangential stress at the interface; normal velocity at the interface matches with the rate of displacement of the interface: $\partial \zeta / 0 t-$ $\left.v_{s}\right|_{\text {rus; }}$ finite pressure and velocities at the drop center as well as at infinity. When the drop center moves, the origin of the coordinates moves with it. Consequently, it seems that the surrounding fluid moves while the drop position keeps unchanged. In the normal stress balance, $T_{s}$ is surface stress including 
the effects of surface rension and surface charge.

$$
\tau_{s}=\sigma\left(\frac{1}{R_{i}}+\frac{1}{R_{2}}\right) \cdots
$$

where 0 is interfacial tension, $R_{1}$ and $R_{2}$ are principal radif of curvature of the drop surface. T. is stress produced by electric charge on the interface.

For free oscillations of a drop with net charge on its surface, the primary effect of net charge on the oscillations is induced by redistribution of the charge on deformed drop surface. A first order perturbation model (weighted in terms of deformation amplitude) is necessary to describe this charge redistribution (Rayleigh 1882, Hendricks and Schneider 1963). When an external altemating electric field exists, direct interaction between the charge and the field is the primary effect on drop motion and oscillations. This effect can be described by a zero order perturbation model. The effect of charge redistribution on the deformed surface becomes secondary. Under ordinary conditions, the secondary effect is much smallex than the primary effect. Only the primary effect will be considered in this paper, therefore, a zero order perturbation model is used. Theoretically, the boundary conditions should be satisfied on the deformed interface. As a zero order perturbation model, the boundary conditions will be satisfied on the undeformed spherical interface, and the normal and tangential components of vectors will be replaced by $I$ and components, respectively.

In zero order perturbation, the electric charge distribution for a slightiy deformed drop is approximated by that on a spherical conductor (Reitz 1967). 


$$
q-q_{\text {net }}+3 \epsilon E \cos \theta
$$

where $q_{\text {net }}$ is average net charge density, $t$ is permittivity of the surrounding Iluid, E is field strength, $\theta$ is polar angle measured from the positive direction of the electric field. For an alternating electric field, E $E^{*} \cos \left(\beta_{e} C\right)$, where $E^{\circ}$ is anplitude and $\beta_{0}$ is frequency of the electric field. The normal stress generated by the charge is

$$
\tau_{e}-\frac{q^{2}}{2 \epsilon}-\left(\frac{q_{n e t}^{2}}{2 \epsilon}+\frac{3}{2}-\epsilon E^{2}\right)+3 q_{n e t} E^{\circ} \cos \left(\beta_{4} t\right) p_{1}+\frac{3}{2}-\epsilon E^{\cdot 2}\left[\cos \left(2 \beta_{0} \tau\right)+I\right] P_{2}
$$

where $P_{n}-P_{n}(\cos \theta)$, is a nth order Legendre function. There is no electric tangential stress on a conducting drop surface (Taylor 1966). The terms in the first parenthesis represent average pressure produced by the net and induced charge. For incompressible fluid, this average pressure only alters pressure distribution but does not contribute to any drop movement. The second and third terms contain only first and second order Legendre functions, respectively. According to the linear oscillation theory (Lamb 1945), the above electric forces will. only affect the first mode (linear translation) and the second mode (prolate-oblate oscillation) motions, respectively. Oscillation frequencies are $\beta_{e}$ and $2 \beta_{e}$ for the first and second modes, respectively. The timeindependent part in the last term will cause a static prolate deformation.

If higher order corrections were included in the model, secondary effects of drop deformation would induce oscillations of some other modes, but amplitudes of those oscillations would be much smallex than the primary motions considered here, as long as the assumpticn of small amplitude is satisfied. 
Solution procedure for the above equations is similar to that described by Chandrasekhar (1961). The expressions of the solution are in complex forms, which involve half order Bessel and Hankel functions.

$$
\begin{aligned}
& p_{1}-p_{1}^{0}+\rho_{1} \sum_{n=1}^{2} a_{1 n} \exp \left(-\omega_{n} t\right) I^{n} p_{n} \\
& P_{0}-P_{0}^{0}+\rho_{0} \sum_{n=1}^{2} a_{2 n} \exp \left(-\omega_{n} \tau\right) \tau^{-n-1} P_{n} \\
& v_{r i}=\sum_{n=1}^{2} \exp \left(-\omega_{n} \tau\right)\left[\frac{a_{1 n} n r^{n-1}}{\omega_{n}}+\frac{a_{3 n}}{r^{3 / 2}} J_{n+1}\left(x_{i}\right)\right] P_{n} \\
& v_{r=0}-\sum_{n=1}^{2} \exp \left(-\omega_{n} t\right)\left[-\frac{a_{2 n}(n+1)}{\omega_{n} r^{n+2}}+\frac{a_{4 n}}{r^{3 / 2}} H_{n+1}\left(x_{0}\right)\right] P_{n}+\frac{a_{52}}{\omega_{1}} \exp \left(-\omega_{1} t\right) P_{1} \\
& v_{\theta i}-\sum_{n=1}^{2} \exp \left(-\omega_{n} t\right)\left(\frac{a_{1 n} r^{n-1}}{\omega_{n}}+\frac{a_{3 n}}{r^{3 / 2}}\left[\frac{J_{n+1}\left(x_{1}\right)}{n}-\frac{x_{1} J_{n+\frac{1}{2}}\left(x_{i}\right)}{n(n+1)}\right]\right) \frac{d P_{n}}{d \theta} \\
& v_{60}-\sum_{n=1}^{2} \exp \left(-\omega_{n} t\right)\left(\frac{a_{2 n}}{\omega_{r} r^{n+2}}+\frac{a_{4 n}}{r^{3 / 2}}\left\{\frac{H_{n+\frac{1}{1}}\left(x_{0}\right)}{n} \cdot \frac{x_{n} H_{n+\frac{3}{2}}\left(x_{0}\right)}{n(n+1)}\right] 1 \frac{d P_{n}}{d \theta}+\frac{a_{51}}{\omega_{i}} \exp \left(-\omega_{1} \tau\right) \frac{d P}{d \theta}\right. \\
& \zeta-R+\left[a_{52} \exp \left(-\omega_{2} t\right)+b_{2}\right] P_{2}
\end{aligned}
$$

Where $p$ and $p^{*}$ are pressure and its time-independent part, respectively; $v$ is velocity; subscripts $i$ and $o$ denote inner and outer fluids, subscripts $x$ and $f$ denote $I$ and $\theta$ components, Iespectively; $n$ is mode number; $\omega_{n}$ tins, is a complex number corresponding to oscillation frequency of nth mode; $x-\sqrt{\omega_{r} / \nu r}$ : $a_{k n}(k=1,2,3,4,5)$ and $b_{2}$ are unknown constants; $J_{n+i}$ and $H_{n+k}$ are half order 
Bessel and Hankel functions of the first kind, respectively; 5 represents deformed drop surface; $R$ is the undeformed spherical drop radius. The last terms in Equations (10) and (12) represent fluid velocity far from the drop relative to the drop center.

Bec ause the problem is linear, we can use the complex form in the boundary conditions and find out the unknown constants numerically, then take the real parts of the results at the end of the calculations. Substituting the above expressions into the boundary conditions and after sone algebraic manipulation, a system of linear algebraic equations for each oscillation mode can be obtained. The equations can be solved numerically with the Gauss elinaination method to get values of the unknown constants.

It is more efficient to present the results in dimensionless form. The variables are nondimensionalized as follow.

$$
r^{*}-I / R, v^{*}=v /\left(\beta_{e} R\right), a^{*}-\left|a_{5 n}\right| / R, P^{*}-P /\left(f_{:} \beta_{e}{ }^{2} R^{2}\right), \psi^{*}=\psi /\left(\beta_{e} R^{3}\right)
$$

where $r, v, a, p$, and $\psi$ are radial coordinate, velocity, amplitude, pressure, and streamfunction, respectively; the superscript * denotes dimensionless variables. Dimensionless groups that affect the dimensionless solution of the second mode oscillation are

$$
e_{1}, e_{0}, \beta^{*}, \rho_{0} / \rho_{1}, f
$$

where $e_{1}-\nu_{i} \sqrt{\rho_{i} / O R}$ and $e_{0}-\nu_{0} \sqrt{\rho_{0} / \sigma R}$, may be regarded as dimensionless viscosities; $\beta^{*}-\beta_{0} \sqrt{\rho_{i} R^{3} / \sigma}$, is a limensionless frequency; $\rho_{0} / \rho_{i}$ is the density ratio: $f-3 \epsilon E^{\bullet 2} R / 2 \sigma$ is the ratio of electric stress to the surface tension 
stress. $f-0.1$ is used in this paper. The time-independent part of the normal stress balance gives solution for $b_{2}:\left(b_{2} / R\right)-f / 4$.

\section{Results and Discussion (for Second Mode)}

Although first mode motion is also oscillatory and may play an important role in enhancing transport processes, it only involves linear translation but no shape deformation. Solution to the first mode can be obtained by the same method used for the second mode. The following discussions are emphasized on the second mode shape oscillation.

Frequency scanning response and resonant frequency

Amplitudes of forced oscillations are functions of the electric field and properties of the fluid system. The linear theory predicts that the amplitude is proportional to the external force. Effects of other parameters are complicated. By measuring the oscillation amplitude while varying the frequency of the force, a scanning frequency response curve can be obtained. Figure 1 shows calculated response curves. The dinensionless amplitude $a^{*}$ is proportional to $f$, and only its relative significance is needed here, therefore, its scale is arbitrary. All the curves in Figure 1 have the same value when $\beta^{*}$ approaches zero. At this point, the drop is in a quasi-equilibrium state and the only parameter that affects $a^{*}$ is $f$. Therefore, a constant $f$ gives a constant $a^{*}$. When $\beta^{*}$ approaches $\infty_{,} a^{*}$ approaches zero. In between, the curve may pass through a peak, which indicates resonance. The shape of the peak is determined by $e_{i}, e_{0}$ and $\rho_{o} / \rho_{i}$. When $e_{i}$ or $e_{0}$ increases, the response curve 
becomes flattened and the dimensionless resonant frequency decreases. When $\rho_{0} / \rho_{1}$ increases, the dimensionless resonant frequency decreases, but the resonant amplitude first decreases then increases. Figure 2 compares a predicted scanning frequency response curve with that measured by Trinh et al. (1982) for a drop driven by an acoustic force. The predicted resonant frequency is tuned to coincide with the measured one by adjusting the interfacial tension (caleulated at $0.029 \mathrm{~N} / \mathrm{m}$ compared to $0.035-0.04 \mathrm{~N} / \mathrm{m}$ given in their paper). The shapes of the curves are similar, indicating that electric force and acoustic force have similar effects on drop deformation.

The resonant frequencies can be obtained from the frequency response curves. Figure 3 compares the resonant frequencies calculated by this model with the free oscillation frequencies (Prosperetti 1980). When calculating dimensionless frequency for free oscillation, the oscillation frequency should be divided by the mode number in order to be consistent with the definition for the forced oscillation. The effects of viscosities on free and resonant frequencies are similar. As viscosities increase, the frequencies decrease. For irviscid fluids, the resonant frequency coincides with the natural frequency. For viscous fluids, the resonant frequency is always smaller than the free oscillation frequency. The larger the viscosities, the greater the difference between then.

Velocity distribution

Variations of velocities in the $\theta$ direction are described by Legendre functions. Only the variations in the r direction will be discussed. For. 
convenience, $\theta-0$ is chosen for $v_{z}$ " and $\theta-\pi / 4$ for $v_{\theta}^{*}$ because in these directions the velocities are maximum. Other factors that affect the velocities are $e_{0}, t_{i}, \rho_{d} / \rho_{i}, \beta^{*}$, and time. It is clear from the velocity expressions that the velocities change with time periodically. The effect of $\beta^{*}$ on the velocities is similar to that on the amplitude. Therefore, these two factors will not be further discussed. In the following, the resonant frequency and the time for maximum radial velocity at the interface are used. Figures 4 and 5 show the radial and tangential velocity profiles with $e_{i}-e_{0}-e$ as a parameter. The velocities decrease with increasing e. When e is small, the drop phase velocities are proportional to the radius except near the interface and the tangential velocity has very large gradients on both sides of the incerface. The gradients increase with decreasing e, but no slip of velocity occurs as long as e is not zero. Consequently, the viscous dissipation is concentrated in a thin boundary layer near the interface for small. viscosity fluids, as has been predicted by Miller and Scriven (1968) for a Eree oscillating drop. If $e_{\downarrow}$ or $e_{0}$ changes individually, the velocity gradient in the corresponding side of the interface will be mostly affected.

Effect of density ratio on the tangential velocity is illustrated in Figure 6 for $e_{i}=e_{0}=0.001$. Of the profiles shown in the Eigure, $\rho_{0} / \rho_{i}-1$ gives the smallest velocity. The velocity profiles shift outward if $\rho_{o} / \rho_{i}<I$, and inward if $\rho_{0} / \rho_{1}>1$. In either of the cases, the larger tangential velocity gradient is always in the less dense side of the interface. 


\section{streamlines}

Since the flow field of the oscillating drop is axisymetric, the strean functions can be easily obtained from the integration of the velocity expressions. Flow pattems for mode 2 are illustrated in Figure 7 . The effects of viscosity and dersity ratio are 1llustrated in dfferent quadrants. The circle in the tiddle indicates the interface of the drop. In ald the conditions the internal and externsl streashines meet at the incerface and form closed cycles. During oscillation the fluids move back and forth once in a period along the streamlines. The centers of the cycles indicate the positions of stagnant rings where the radial and tangential, velocities are zero. The 6 position of the rings are detaroined by $v_{z}-0$, which glves $\theta-\cos ^{-1} \sqrt{1 / 3}$. $v_{\theta}-$ 0 deternines the radial position of the rings, which curns out to be a function of many parayeters. When the denstifes ar"e the same ( (a) and (b)), the stagnant rings move to the less viscous side of the interface, while when the viscous effects are the same ( $(a),(c)$ and $(d))$, the rings move to the less dense stide of the interface. The internal streamlines observed by Trinh et al. (1982) qualitatively agtee with the predicted results,

\section{Conclustions}

The proposed osciliation model for a drop in an alcernating electric field is able to predict velocity field, scanning frequency response curve, resonant frequency, and streamlines. The predicted scanning frequency response curve and the streamlines are in good agreement with experimental observations. It is found that the resonant frequency of the forced oscillation is equal to the fre- 
oscillation frequency for Inviscid Eluids, but always smaller than the free oscillation frequency for viscous fluids. The viscosities and the density ratio have significant influence on the velocity profiles.

The enhancement of a transport process by an alternating electic field depends on how mach the systems are disturbed. Operation at a resonant frequency is desirable because it produces maximu disturbance for the same energy input. The predicted scanning frequency response curves can give best operation frequency range for any fluid system. The velocity distribution is very useful for the modelling of a mass or heat transfer process.

\section{Aciknowlejgment}

This work was supported by the Division of Chernical Science, Office of Basic Energy Science, Office of Energy Research, U, S. Department of Energy under contract FG07-86ER.13572.

Notation

$$
\begin{aligned}
& a^{*} \text { - dimensionless oscillation amplitude } \\
& a_{k n} \text { - constants in expressions of solutions, } k-1,2,3,4,5 \\
& b_{2} \text { - second mode static deformation } \\
& E, E^{*} \text { - alternating electric field strength, amplitude of } E, \forall / m \\
& e-v \sqrt{\rho / a R} \\
& E^{*}-3 \in E^{\circ 2} R /(2 \sigma) \\
& H_{n+4}-\text { half order Hankel Eunction }
\end{aligned}
$$




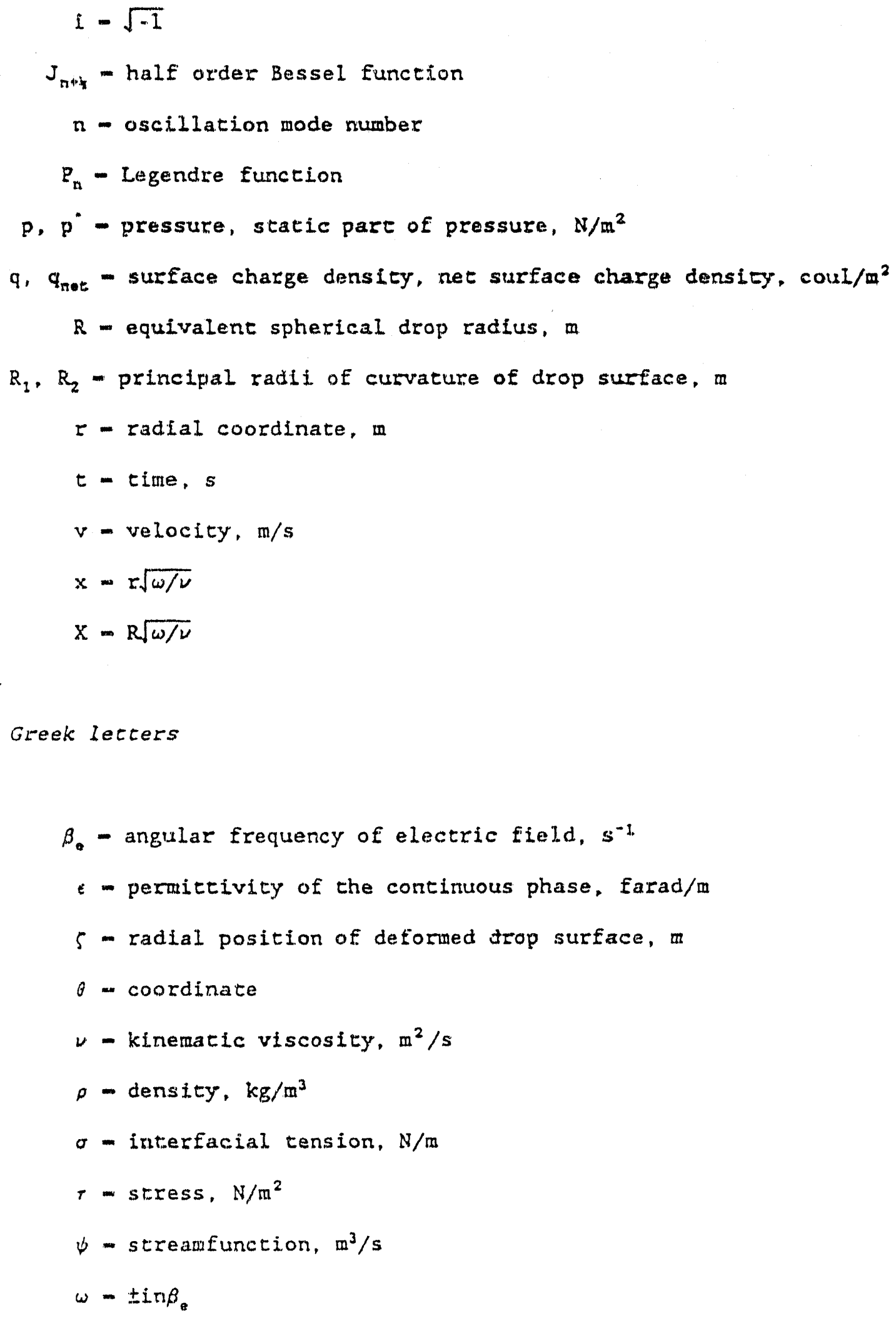


Subseripes
e - electric field
$i$ - Inside the drop
$n-1,2$, oscillation mode number
- outside the drop
r - radial direction
s - on the interface
$\theta-\theta$ direction

Superscripts

* - dimensionless variables

\section{Literature cited}

Chandrasekhar, S., Hydrodynamic and Bydromagnetic Stability, Oxford at the Clarendon Press (1961).

Hendricks, C. D. and J. M. Schneider, "Stability of a Conducting Droplet under Influence of Surface Tensio and Electric charge," Am. J. Phys., 31, 450 (1963).

Kaji, N., Y. H. Mori. Y. Tochitani, and K. Komotori, "Augmentation of DirectContact Heat Transfer to Drops with an Intermittent Electric Field," J. Heat Transfer, 102, $32(1980)$. 
Ka:-, N., Y. H. Mori, and Y. Tochitani, "Heat Transfer due to Electrically Induced Resonant Oscillation of Drops," J. Heat Transfer, 107, 788 (1985).

Kawalski W. and Z. Ziolkowski. "Increase in Rate of Mass Transfer in Extraction Columns by Means of an Electric Field," Intermational Chem. Eng., 21(2), 323 (1981).

Lanb, H., Rydrodynamies, 6th edition, 1st USA edition, Dover, New York (1945).

Levich, V. G.. Physicochemical Rydrodymamics, Prentice-Hal.I, Englewood Cliffs, N. J. (1962).

Marstor, P. L., "Shape Oscillation and Static Deformation of Drops and Bubbles Driven by Modulated Radiation Stresses-Theory," J. Acoust. Soc. Am., 67(1), $15(1980)$.

Miller C. A. and L. E. Scriven, "The Oscillations of a Fluid Droplet Immersed in Another Fluid," J. Fluid Mech., 32, Part 3, 417 (1968).

Prosperetti, A., "Normal-Mode Analysis for the Oscillations of a Viscous Liquid Drop in an Immisctble Liquid," J. Méc., 19, 149 (1980).

Rayleigh, Lord, "On the Equilibrium of Liquid Conducting Masses Charged with Electricity," Phil. Mag., 14, 184 (1882). 
Reftz, J.R. and F. J. Milford, Foundations of Electromagnetic Theory, 2nd edition, Addison-Wesley, Reading Massachusetts (1967).

Sozou, C., "Electrohydrodynamics of a Liquid Drop: The Time-Dependent Problem," Proc. R. Soc. Lond., A331, 263 (1972).

Taylor, G., "Studies in Electrohydrodymamics I. The Circulation Produced in a Drop by an Electric Field," Proc. R. Soc. Lond., A291, 159 (1966).

Thornton, J. D., "The Applications of Electrical Energy to Chemical and Physical Rate Processes," Rev. Pure and Appl. Chem., 18, 197 (1968).

Torza, S., R. G. Cox, and S. G. Mason, "Electrohydrodynamic Deformation and Burst of Liquid Drops," Phil. Trans. R. Soc. Lond. A269, 295 (1971).

Trinh, E., A. Zwern, and T. G. Wang, "An Experimental Study of Small-Amplitude Drop Osci.llations in Immiscible Liquid Systens," J. Fluid Mech., 115, 453 (1982). 


\section{Figure Captions}

Figure 1. Predicted scanning frequency response curves for 2 nd mode oscillation. The line passing the peaks of the response curves indicates the effect of densicy ratio on the resonant amplitude for $e_{1}-e_{0}-0.01$.

Figure 2. Comparison of predicted and observed (Trinh et al. 1982) 2nd mode scanning frequency response curves for a silicone/CCI $1_{4}$ drop immersed in distilled water. The smooth curve is predicted. Drop volume $-1.5 \mathrm{~cm}^{3}, \rho_{1}-$ $\rho_{0}-990 \mathrm{~kg} / \mathrm{m}^{3}, \nu_{1}-3.2 \times 10^{-6} \mathrm{~m}^{2} / \mathrm{s}, \nu_{0}-1.01 \times 10^{-6} \mathrm{~m}^{2} / \mathrm{s}$.

Figure 3. Comparison of resonant frequency of forced oscillation and free oscillation frequency for 2 nd mode.

Figure 4. Radial velocity distribution for a drop undergoing 2 nd mode oscillation with $e_{1}-e_{0}-e$ as a parameter.

Figure 5. Tangential velocity distribution for a drop undergoing 2 nd mode oscillation under the same conditions as those of Figure 4.

Figure 6. Tangential velocity distribution for a drop undergoing 2 nd mode oscillation with $\rho_{0} / \rho_{i}$ as a parameter.

Figure 7. Streamlines for a drop undergoing 2 nd mode oscillation.
(a): $e_{1}-e_{0}=0.001, p_{0} / p_{1}=1$;
(b) : $\mathbf{e}_{1}-0.01, \mathbf{e}_{0}=0.001, \rho_{0} / \rho_{1}=1$;
(c): $e_{1}-e_{0}-0.001, p_{0} / p_{2}-100$;
(d): $e_{1}=e_{0}=0.001, \rho_{0} / \rho_{1}=0.01$. 


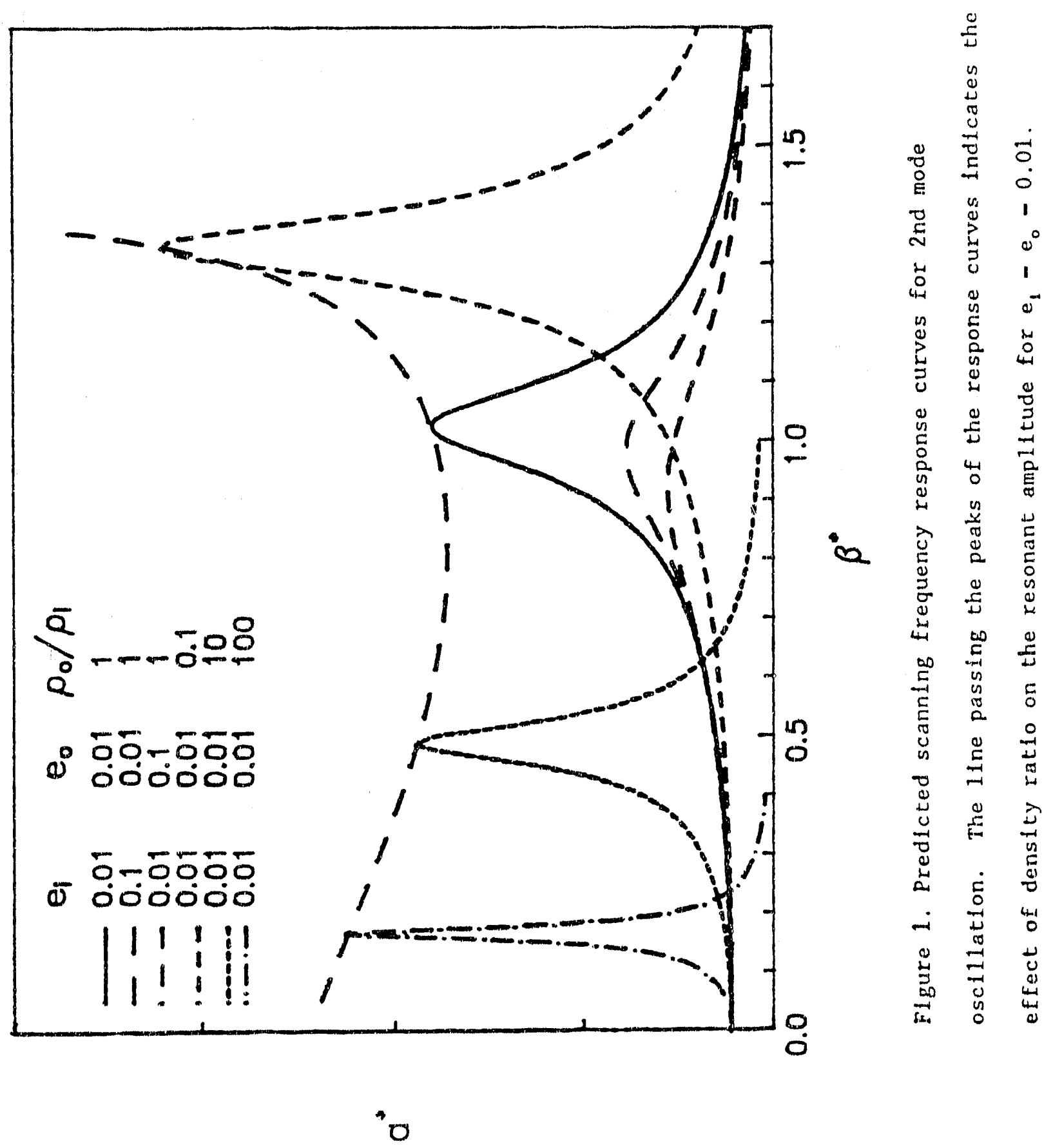




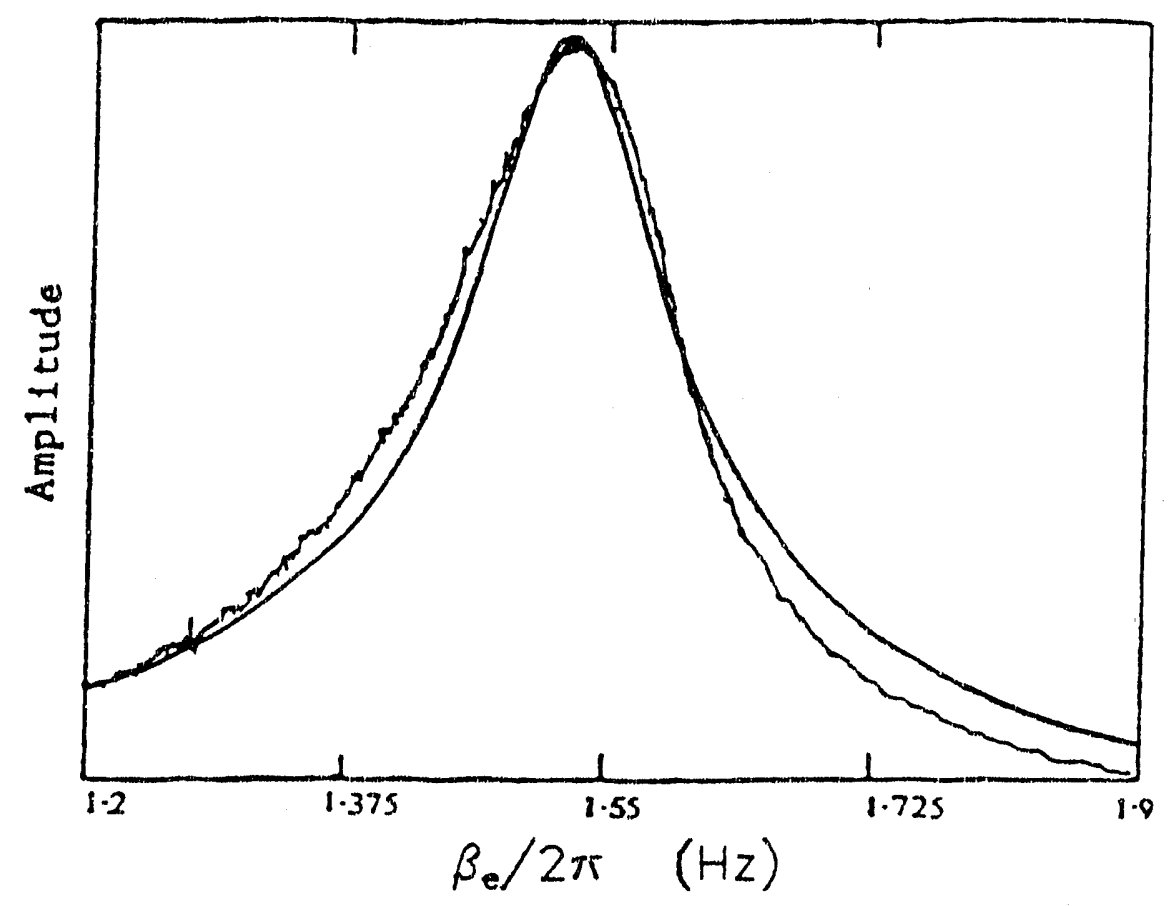

Figure 2. Comparison of predicted and observed (Trinh et al. 1982) 2nd mode scanning frequency response curves for a silicone/CCl 4 drop immersed in distilled water. The smooth curve is predicted. Drop volume $-1.5 \mathrm{~cm}^{3}, \rho_{1}-$ $\rho_{0}=990 \mathrm{~kg} / \mathrm{m}^{3}, \nu_{1}-3.2 \times 10^{-6} \mathrm{~m}^{2} / \mathrm{s}, \nu_{0}-1.01 \times 10^{-6} \mathrm{~m}^{2} / \mathrm{s}$. 


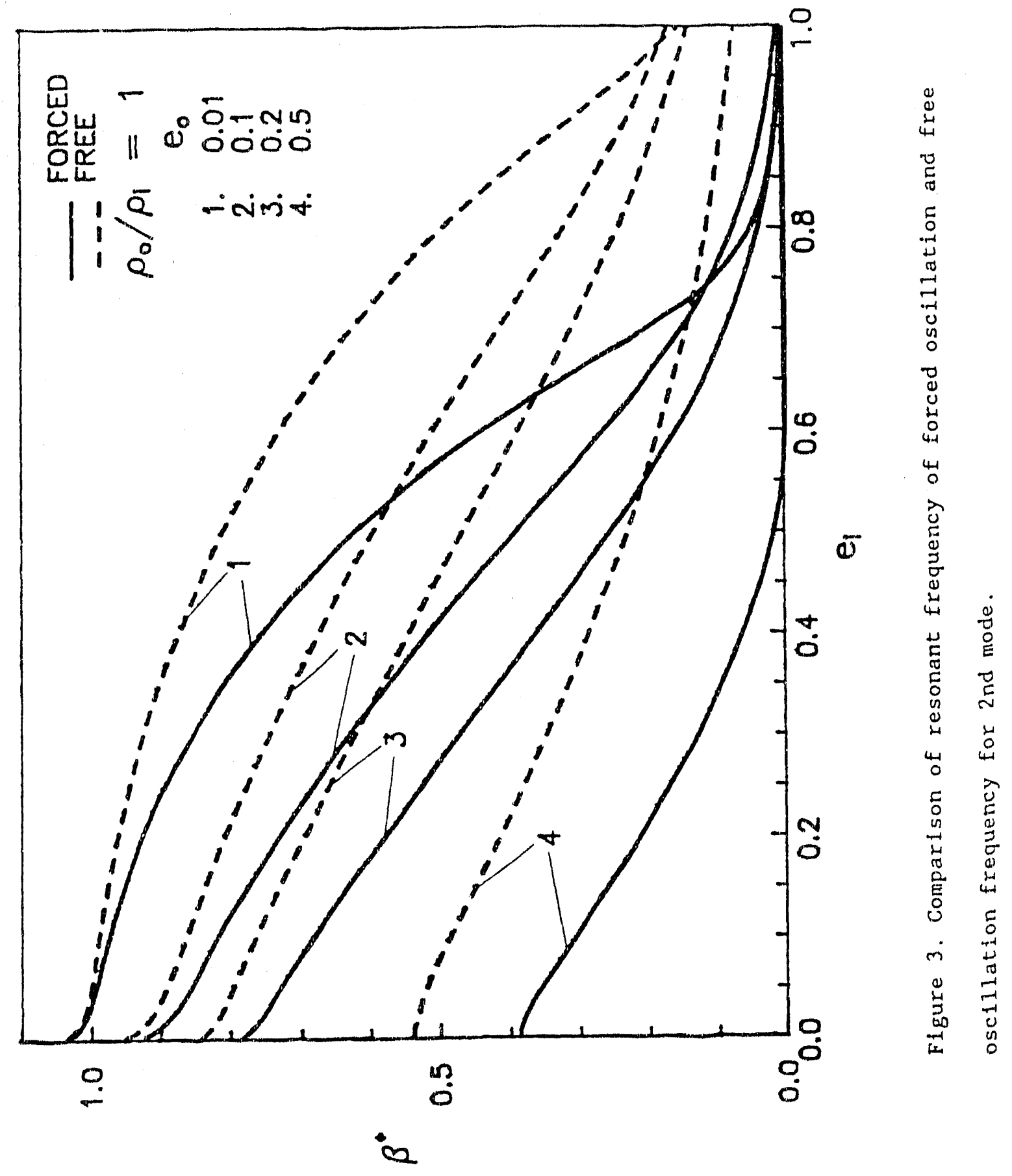




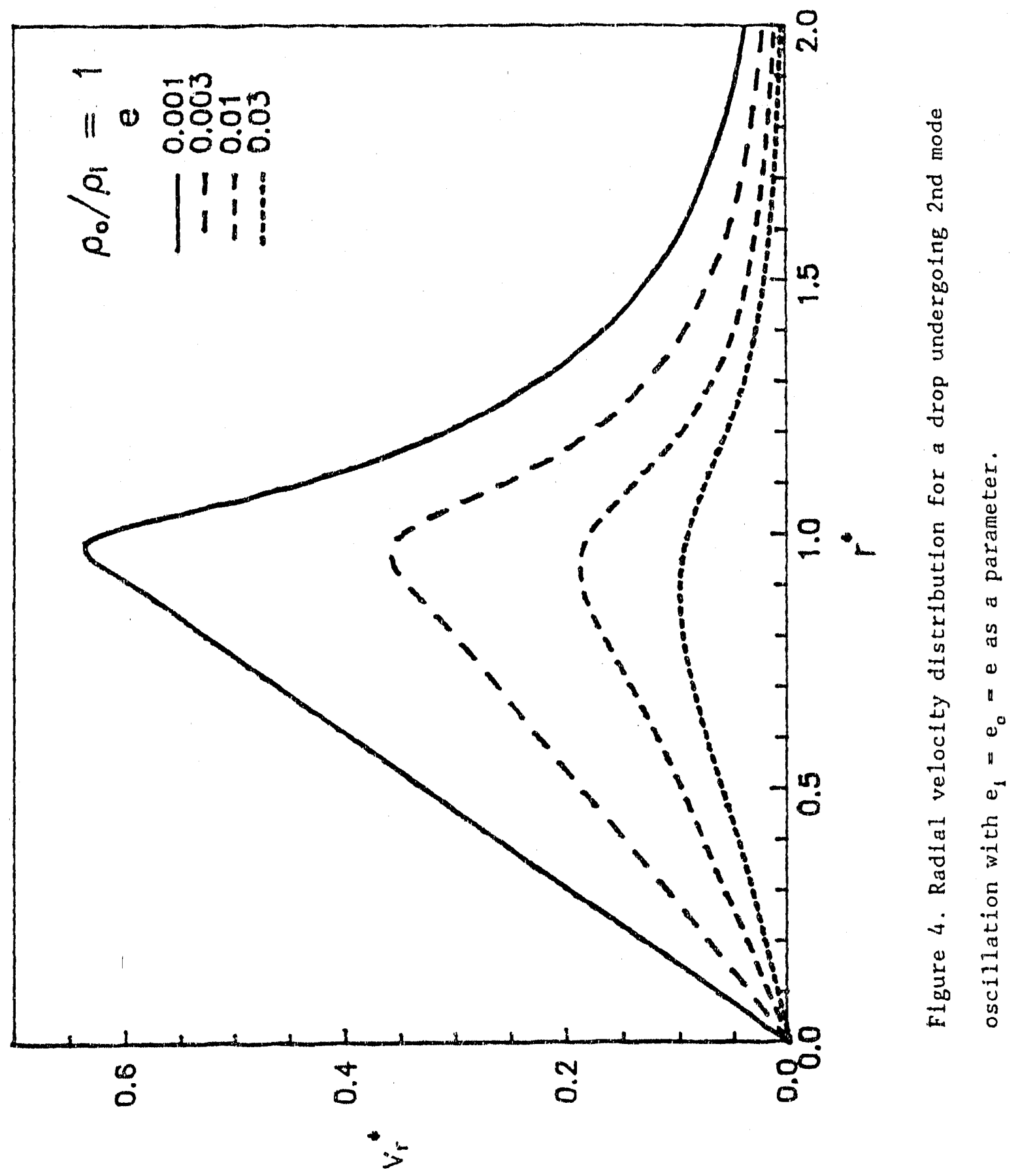




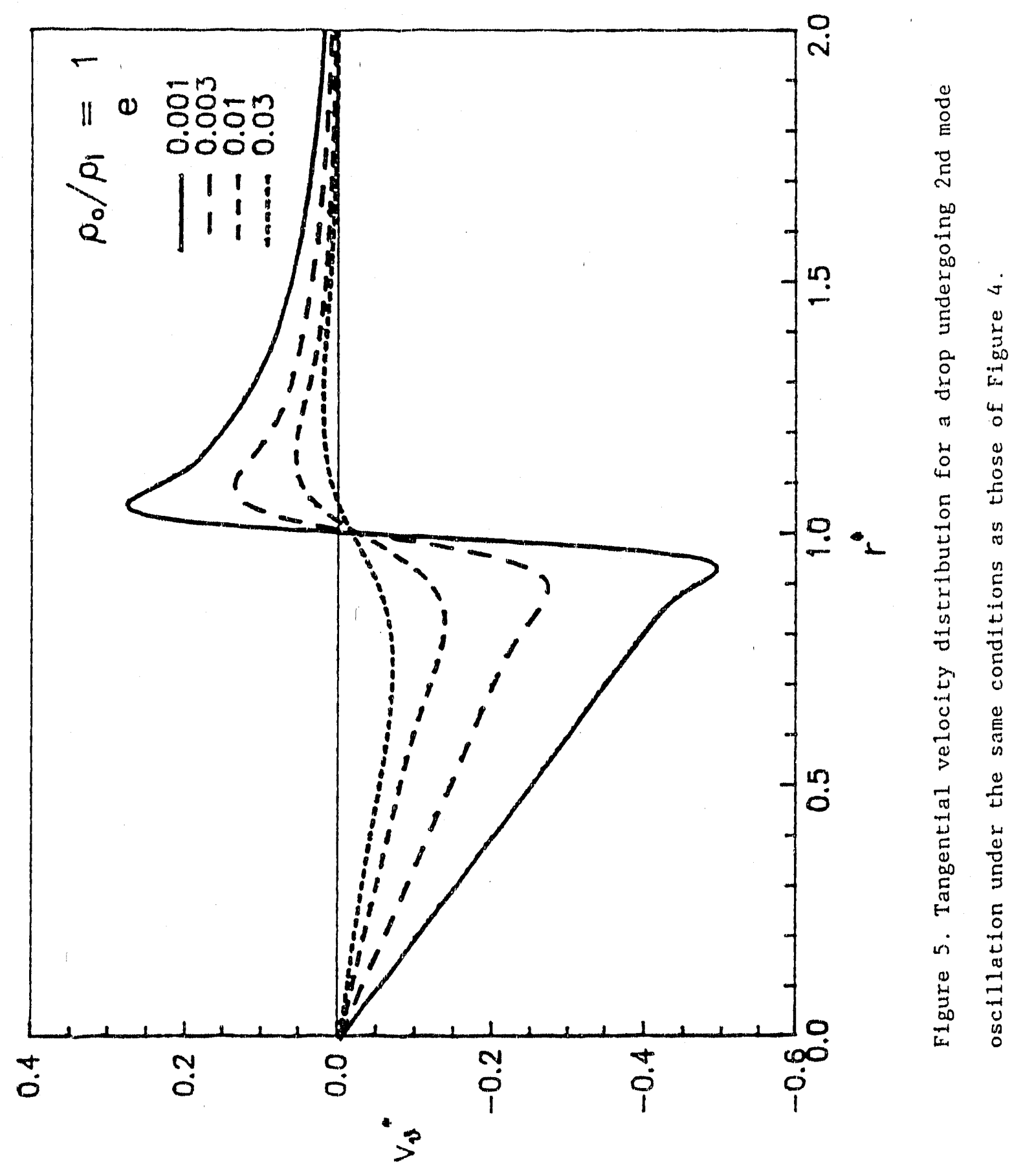




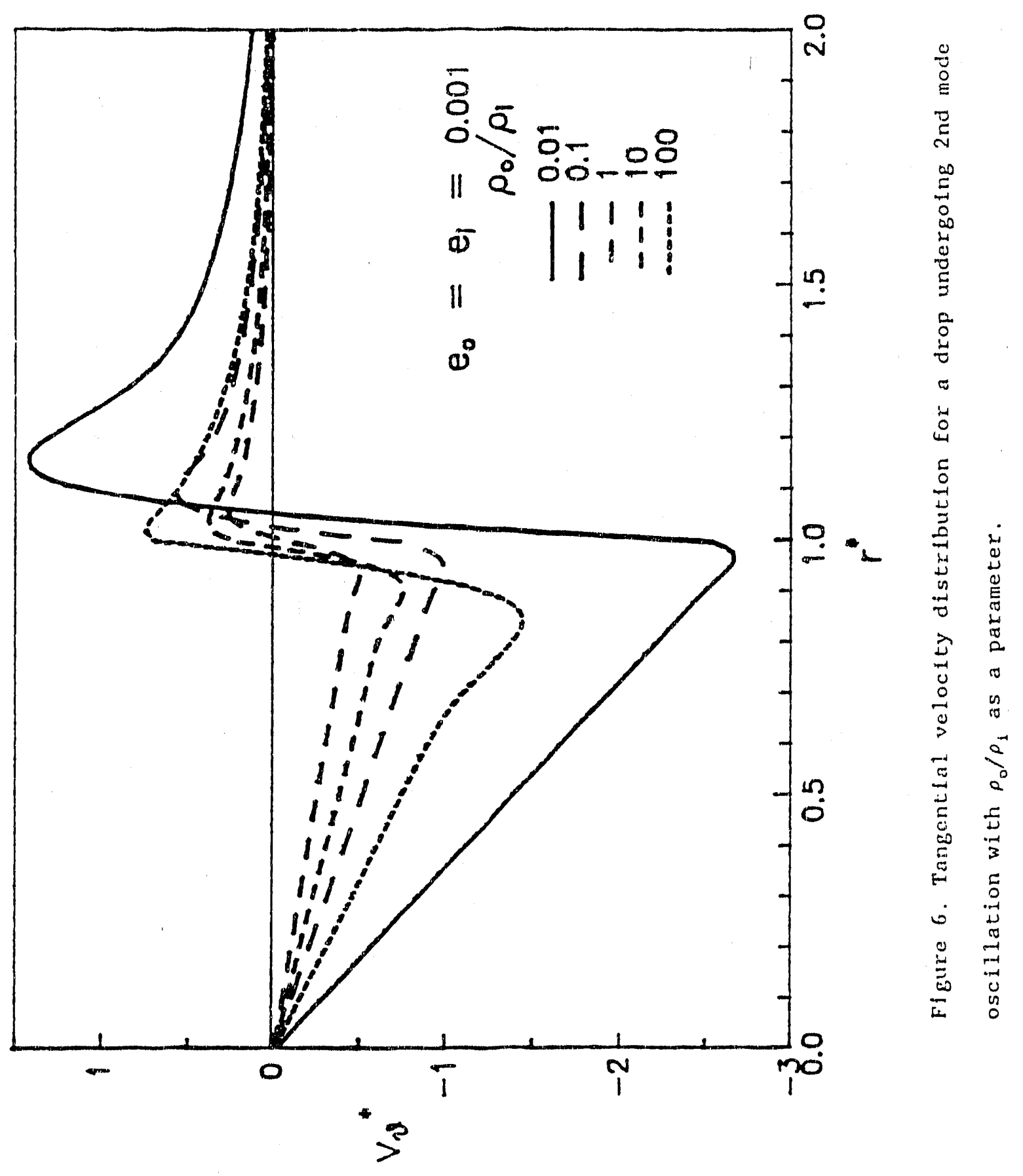




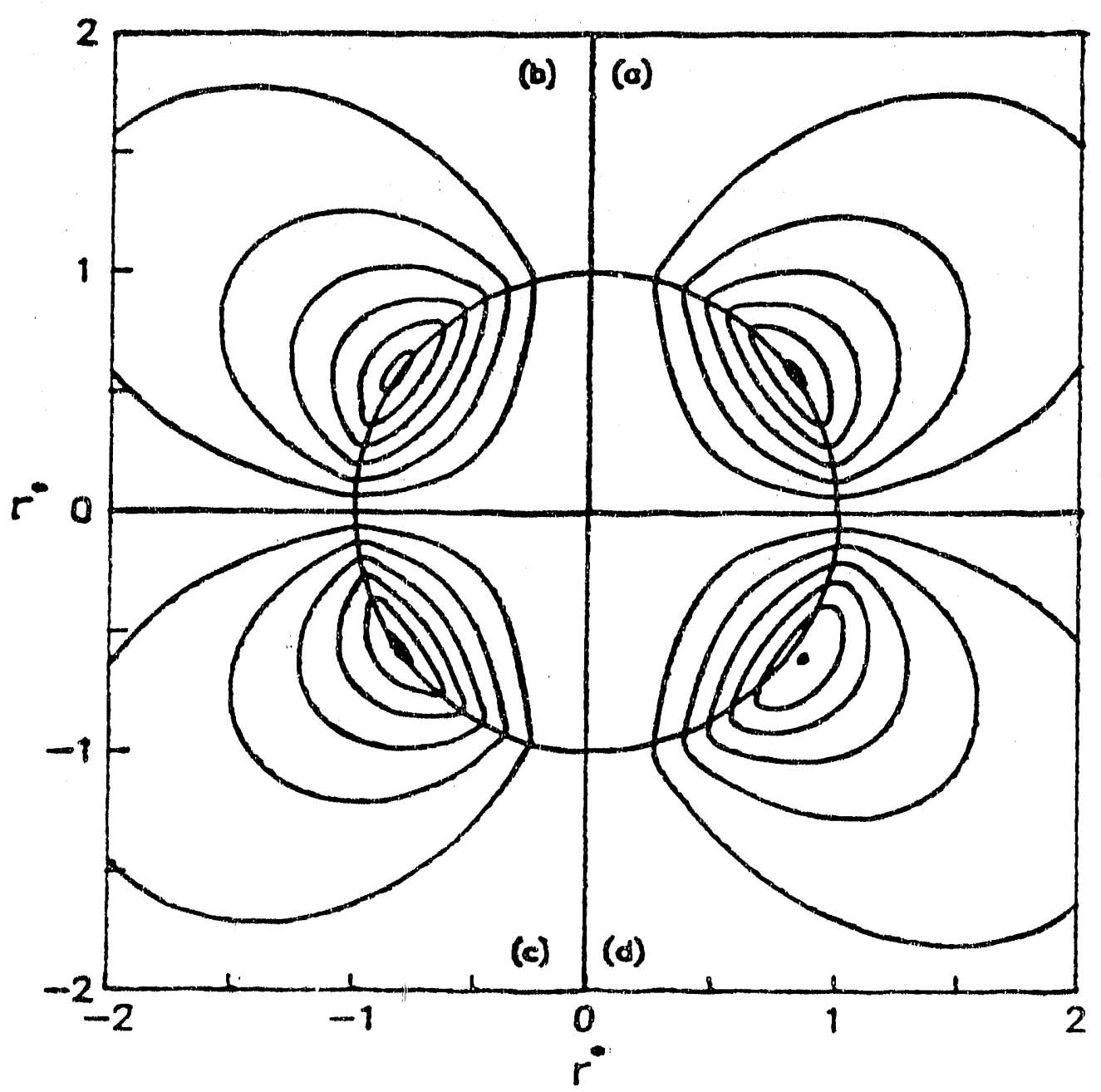

Figure 7. Streamlines for a drop undergoing 2 nd mode oscillation.

(a) : $e_{1}=e_{0}=0.001, p_{0} / \rho_{1}=1 ;$ (b): $e_{1}=0.01, e_{0}=0.001, \rho_{0} / p_{i}=1$;

(c) : $e_{i}=e_{0}=0.001, \rho_{0} / p_{i}=100 ;$ (d): $e_{i}=e_{0}=0.001, \rho_{0} / \rho_{i}=0.01$. 
